\title{
Preservación de la cultura, clima, identidad e imagen en la empresa ecuatoriana
}

\section{Maintaining the culture, climate, identity and image in the Ecuadorian business}

Rubén Dario Ramos Grijalva ${ }^{1}$, Wilson German Nieto Aguilar ${ }^{2}$, Alex Vinicio Zapata Álvarez ${ }^{3}$

INFORMACIÓN DEL

\section{ARTÍCULO}

Fecha de recepción: 27 de Julio de 2021.

Fecha de aceptación: 30 de Octubre de 2021

$\overline{1}$ Doctor en Comunicación, UNIACC. Docente-investigador, Universidad San Francisco de Quito - Ecuador. E-mail: rramosg@asig.com.ec Código ORCID:

https://orcid.org/0000-0003-2611-8579

Máster en Marketing, Universidad Autónoma de Madrid. Docenteinvestigador, Universidad Tecnológica Israel - Ecuador

E-mail: wnieto@uisrael.edu.ec

Código ORCID:

https://orcid.org/0000-0003-3971-5479

3 Magíster en Arquitectura de la Información, PUCE. Docenteinvestigador, Instituto Superior Tecnológico Vicente León - Ecuador

E-mail: a.zapata@istvicenteleon.edu.ec Código ORCID:

https://orcid.org/0000-0003-1952-0447

CITACIÓN: Ramos, R.D., Nieto, J., \& Zapata A. (2021). Preservación de la cultura, clima, identidad e imagen en la empresa ecuatoriana. Podium, 40, $75-88$.

doi:10.31095/podium.2021.40.5

ENLACE DOI:

http://dx.doi.org/10.31095/podium.202 1.40 .5

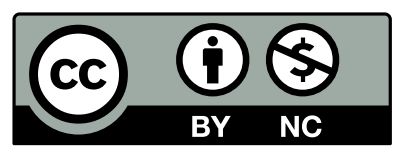

\section{Resumen}

El preservar la cultura, el clima, la identidad e imagen, es decir activos intangibles, es un modelo de gestión empresarial vigente desde mediados del siglo pasado. Su accionar se invisibiliza por el error frecuente en las organizaciones que enfatizan exageradamente los activos tangibles. Partiendo de una metodología cuantitativa, se caracteriza la investigación como descriptiva no experimental transversal; y si bien existen publicaciones similares en Ecuador en los dos últimos años, conforme una indagación en Google Académico, estas distan de la presente en que redundan en análisis sin enfoque sinérgico. En este estudio se pretende verificar en la población seleccionada, el grado de preservación, difusión y satisfacción respecto del esfuerzo que hacen las empresas por gestionarlos. Los resultados muestran la incipiente disposición por preservar los activos intangibles y se establecen conclusiones centradas en la necesidad de crear conciencia para la diligencia de estos.

\section{Palabras Clave:}

Activos intangibles, activos tangibles, cultura, clima, identidad, imagen.

Clasificación JEL: M10, M14, D21.

\begin{abstract}
Maintaining the corporate culture, climate, identity and image in regards to intangible assets, is a business management model that has been used since mid-way through last century. Their actions are rendered invisible by the frequent error in organizations that over emphasize tangible assets. On the basis of a quantitative methodology this research is characterized as descriptive non-cross-sectional experimental; and although there have been similar publications in Ecuador in the last two years, according to an inquiry from Google Scholar, these are far from the present due to the fact that they result in analysis without synergistic approach. This study aims to verify, in the selected population, the level of preservation, spread and satisfaction regarding the companies' efforts to manage intangible assets. Results show a surging willingness to preserve the intangible assets and conclusions are established based on the need of raising awareness for the diligence of them.
\end{abstract}

Keywords:

Intangible assets, tangible assets, culture, climate, identity, image.

JEL Classification: M10, M14, D21. 


\section{Introducción}

La preservación de los activos intangibles frente a los activos tangibles, no guarda la misma relación, pocas son las ocasiones en las que se publican artículos sobre clima, cultura, identidad e imagen en las empresas ecuatorianas. Una de las entidades que propician este tipo de estudios es la Asociación Ecuatoriana de Comunicación Interna del Ecuador - AECI (2019), pero sus estudios no son periódicos. Existen estudios como MERCO (Corresponsables, 2020) que analizan su impacto y certificaciones como la de Great Place to Work (2020) en los que se destacan indicadores de clima y cultura para seleccionar a los mejores sitios para trabajar; pese a ello, no se halla consolidada una cultura de preservación de intangibles. Uno de los planteamientos de este estudio es que esta problemática es poco abordada, a pesar de que mucho se habla en seminarios $y$ en las universidades sobre su estatuto como herramienta de desarrollo empresarial. Asimismo, existen estudios recientes en esta materia sobre temáticas similares en los dos últimos años en Ecuador (Vire, 2019; Paredes y Quiroz, 2021; Montaño, 2019; Falconí, 2020); sin embargo, no hay uno que haga relación a la diligencia sinérgica de: cultura, clima, identidad e imagen.

Es tal la importancia de este tipo de activos que, tanto Ventura (2001) como Scheinsohn (2009), coinciden en que son imprescindibles de ser gestionados. Ventura (2001) los llama sistema débil en contraposición al fuerte o tradicional: marketing, talento humano, producción y finanzas; Scheinsohn (2009) en cambio, los denomina el hexágono de la gestión corporativa. Ambos, con minúsculos diferimientos concuerdan en que los activos intangibles son el clima, la cultura, la identidad e imagen corporativas y los que deriven de su sinergia. Ramos (2012), por su parte plantea la necesidad de establecer una fórmula para el constructo de la imagen global en la que los activos mencionados son prioritarios: " $\mathrm{E}+\mathrm{CYC}+\mathrm{I}+\mathrm{EI}=\mathrm{IG}$. En donde: $\mathrm{E}=$ estructura, $\mathrm{CyC}=$ cultura $\mathrm{y}$ comunicaciones, $\mathrm{I}=$ identidad, $\mathrm{EI}=$ estrategia de identidad e IG=imagen global" (p. 67).

En el contexto empresarial ecuatoriano, existe una concepción esencialmente reduccionista sobre el rol de los intangibles; y en ello radica la justificación de este estudio, en puntualizar su estado actual. Por ello, la necesidad de formularse las siguientes preguntas de investigación: ¿A través de qué mecanismos las organizaciones preservan sus intangibles? ¿A través de qué medios y actividades las organizaciones difunden acciones relacionadas a sus intangibles? ¿Cuál es el grado de satisfacción que representa el esfuerzo en su preservación?

Para responder a estas preguntas, se extrajo una muestra no aleatoria disponible de 400 empresas de distinto giro de negocio a las que se les aplicó un cuestionario de preguntas con respuestas de opción múltiple, con el fin de lograr los siguientes objetivos específicos: 1) Determinar mecanismos de preservación de intangibles, 2) Identificar los medios y actividades a través de los cuales se 
difunden acciones para su difusión y, 3) Establecer la satisfacción respecto del esfuerzo que cada empresa hace.

En este artículo, se acentúa la importancia que adquieren la cultura, el clima, la identidad y la imagen, destacando con ello, el valor agregado que asumiría una empresa reputada; luego, una sólida reputación corporativa es el mejor intangible al que toda organización debe aspirar.

\section{Revisión de literatura}

Para todos quienes están inmersos en el ámbito empresarial, es sabido que los activos intangibles no tienen formas físicas, no son materiales y por tanto no se pueden ver ni tocar (Sevilla, 2015), motivo por el cual, en esta revisión teórica más que definirlos, se analiza la simbiosis que hay entre la cultura, clima, identidad y su aporte al fortalecimiento de la imagen. "A finales de los ochenta toma fuerza el reconocimiento de los elementos intangibles de una organización como fuente de ventaja competitiva debido a que éstos son menos dependientes de la posición en el mercado" (Wernerfelt, 1984, citado en Suárez, Álvarez y Vásquez, 2020, p.150).

\section{Cultura}

La cultura de una organización "es el conjunto de disposiciones inmateriales que dan razón de su actividad, y la comunicación es parte de ella" (Del Pozo, 2000 , p. 29); su relación con el clima, la identidad e imagen es indisoluble. Los valores y las creencias que la conforman son el punto de partida para la consolidación de los intangibles. Todo lo que haga una empresa, aun cuando de ello no se tenga conciencia, se regula y determina por su cultura y tipo. Cuando esta pasa inadvertida, es una cultura de contexto bajo.

La cultura, de acuerdo con esta definición y a la de la mayoría de autores versados en el tema, es la manera en que las empresas se comportan, actúan y manifiestan sobre la base de un marco de referencia común, dentro del cual, la identidad se comunica, el clima se dosifica y la imagen se percibe. La sinergia entre estas referenciará una coherencia entre el ser, hacer y decir de las organizaciones. A la falta de un manejo eficiente de la cultura, el clima queda sujeto a la casualidad, la identidad no será comunicada en su plenitud y la imagen será percibida a medias, tanto a nivel interno como externo. Si para Garrido (2008), la cultura es una construcción comunicacional, entonces su estatuto de transversalidad en el resto de intangibles es un hecho irreversible, aspecto que lo confirma Van Riel (1997), cuando asevera que, junto con la comunicación y el simbolismo, es lo que constituye la identidad de las empresas.

La cultura, de acuerdo con los autores mentados, no puede quedar sobre el papel, vale decir estática e inmóvil, distante del plan de negocios; por el contrario, debe asumir un rol dinamizante y vinculador, es ahí donde el maridaje con las comunicaciones hace posible que sus valores y creencias se transmitan por todo el tejido social de la empresa. 
Metaforizando lo dicho, la cultura pasa a convertirse, sin reverso alguno en su ADN (Ramos, 2019).

\section{Clima}

En las revisiones bibliográficas hechas sobre clima, se encuentran factores que lo modifican, pero muchos autores coinciden en que el factor de más alta ponderación es la cultura. Además, la distinción que algunos autores hacen de la cultura y el clima es imperceptible, parecería que son nociones sinónimas. A pesar de ello, el clima tiene su propia dimensión de creación en tanto intangible. Márquez (2002), plantea que siendo el clima la actitud del colaborador frente a su propio trabajo, este proceder, está basado en las creencias y valores que desarrolla, es decir en la cultura organizacional. Las actitudes son determinadas conjuntamente por las características del marco de referencia y; dentro de este, hasta pueden recibir motivaciones intrínsecas o extrínsecas de recompensa o castigo, para lo cual, sería pertinente aplicar un "test que mida la motivación laboral a nivel extrínseco e intrínseco" (Aguirre, Calvache, y Osejo, 2019, p. 37).

El clima, a diferencia de los otros intangibles se dosifica, no posee un estado puro, lo que significa que depende en primer lugar de la cultura y de la identidad de efecto interno para su consolidación. Su permeabilidad se subordina a la alternancia de dos cuestionamientos. El primero de ellos responde al desarrollo de los colaboradores (Brito, Pitre, y Cardona,
2020); o si, por el contrario, los objetivos de la empresa están por encima de todo; el segundo, tiene que ver con la flexibilidad respecto al entorno. Una cultura abierta o activo adaptativa (Scheinsohn, 1996), permitirá que el clima se gestione de mejor manera, dejando las interrogantes anteriores supeditadas a la cultura y no dependientes del clima per se.

El clima se sujeta a una serie de cuestiones, lo que lleva a la conclusión de que su percepción se forja en las relaciones diádicas, grupales y organizacionales. El clima, en tanto resultado, no puede ser una consecuencia casual, sino más bien causal, toda vez que depende en sumo grado de la convergencia o sinergia con la cultura.

\section{Identidad}

La identidad se forma desde una pregunta que todos deberían hacerse: ¿Quiénes somos? De ahí, parte una serie de concepciones que se han hecho, y se resumen más o menos así: es la esencia de la existencia de la empresa. Etimológicamente, la palabra identidad tiene su origen en el latín ídem, que significa lo mismo. Es decir, son los elementos distintivos que, la hace ser ella y no otra. Garrido (2008), la describe como factor de cohesión al interior del sistema-empresa y de sus posibilidades estratégicas para estimular la tan anhelada diferencia con las demás compañías. Capriotti (1999), cavila desde dos perspectivas: la filosofía y la cultura corporativas. Se deja entrever de nuevo la interrelación indisoluble, de la que ya se 
comentó anteriormente. Se entiende que, la filosofía debe ser consensuada y compartida por todos los colaboradores. Mientras, la cultura que para Van Rekom, Van Riel y Wierenga (1991), es una de las tres maneras en que la identidad se auto presenta, se la acepta y comparte.

Para resumir, Molina (2004), plantea una definición unificadora que sintetiza los aportes de Garrido (2008) y Capriotti (1999) como la suma de la historia, la filosofía y las estrategias de una empresa. No se puede cambiar con facilidad. Sin embargo, está sujeta a evolución. Cuando cambia la identidad también muda la imagen.

\section{Imagen}

La noción imagen, es un término polisémico, además de que posee una naturaleza de análisis multidimensional (De Castro y Navas, 2006). En su estatuto convergen algunos elementos que bien valen ser analizados para determinar el nivel de influencia en su constitución. El reducirla a la percepción de los públicos de interés, tema demasiado trascendental, es verle al fenómeno desde una sola dimensión, cuando también son otros los factores que inciden en su formación.

No hay que perder de vista que, al ser un intangible, su análisis puede someterse a cierta complejidad. Su relación con el clima, cultura e identidad, tiene un alto valor de consideración, y para ser más preciso, este estudio ha venido discutiendo sobre esa interrelación, además de su preservación.
La definición de imagen organizacional con la que se teorizará, es la de un conjunto de significados adaptados a cualquier actividad funcional (marketing, publicidad, relaciones públicas, propaganda, periodismo, diseño gráfico, etc.), y que por medio de las cuales los públicos vinculados llegan a conocerla, describirla, recordarla y relacionarla; teniendo como resultado de esta interacción: creencias, ideas, sentimientos e impresiones. Esa representación mental, es consecuencia de la interpretación de los esfuerzos informativos (Xifra, Ramos, y Enríquez, 2016).

La imagen posee dimensiones: corporativa; productos y servicios; employer branding (clima, cultura, talento humano); e institucional (sociedad, comunidad y sector público: rol social). Estos cuatro niveles, nos lleva a su construcción global o en palabras de Fernández y Alarico (2016, p. 65), “al perfil de imagen real"; la que se presume por sentido común, no puede quedar al libre albedrío; supuesto este que, al parecer algunas empresas omiten más por desconocimiento que por incompetencia de sus expertos en comunicación. No la perciben "como un componente estratégico" (Hinojosa y Cogco, 2020, p. 113). El fortificar los activos tangibles, no es bajo ninguna circunstancia razón para omitir a los intangibles. Ambos sistemas, bien complementados y gestionados, la vigorizarían; demostrando con ello que, "la imagen organizacional es significativa para muchas organizaciones" (Ramos y Valle, 2020, p. 293).

Por añadidura cabe señalar que una 
crisis se presenta e intensifica, al no identificar los acontecimientos previstos e imprevistos que, derivados del entorno pueden afectar a las empresas; de tal manera que, sin importar el alcance de su gravedad, la organización como sistema autopoiético que es, debe hallar la adaptabilidad a sí misma y con sus sistemas circundantes, es decir con sus públicos de interés -suprasistema- y con el macrosistema. Si a tiempo no se identifican los sucesos susceptibles de generar crisis y no se los valora, sino se diseñan acciones táctico-estratégicas y no se cuenta con un mecanismo de evaluación con el fin de medir su impacto; nada raro sería que, en cualquier momento, y de forma súbita la estructura de una empresa se vea afectada, irremediablemente, ya sea por el lado de los tangibles o de los intangibles.

\section{Metodología}

En esta investigación no experimental y de corte transversal en su fase empírica, se solicitó la participación de algunas organizaciones de distintos sectores y de diversas actividades. Considerando el confinamiento y aprovechando la eficiencia de las tecnologías de transferencia de contenidos e información, además del tiempo y el costo, se elaboró una encuesta online para una muestra no probabilística a empresas ubicadas en el Distrito Metropolitano de la ciudad de Quito.

Las unidades de análisis se ubican en distintos giros de negocio: alojamiento, servicios de comida, salud, asistencia social, financieras, de seguros, públicas, planes de seguridad, agricultura, ganadería, entretenimiento, recreación, construcción, enseñanza, etc., que de acuerdo a un criterio de muestreo no probabilístico por conveniencia, se escogieron a 400 empresas registradas en la base de datos de la Cámara de Comercio de Quito.

Se diseñó un cuestionario de 20 preguntas de respuesta con opción múltiple en la herramienta Google Form. En primer lugar, se procedió a establecer preguntas de información preliminar y general como el nombre de la empresa (opcional), actividad a la que se dedica, volumen de ventas anuales y alcance (local, cantonal, provincial, nacional o multinacional). En los ítems relacionados a la satisfacción con respecto de la preservación del clima, la cultura, la identidad y la imagen se elaboró una escala de Likert de 0 a 10 , siendo 0 , totalmente insatisfecho, y 10 , totalmente satisfecho.

El instrumento fue validado por expertos en comunicación organizacional y en investigación de mercados. Se sometió el cuestionario a un mismo grupo de 10 personas en dos ocasiones distintas y con el método test - retest, $r$ de Pearson, se encontró que el instrumento arrojó un coeficiente de correlación de estimación de confiabilidad de 0,92. Quienes respondieron el cuestionario fueron directores $\mathrm{y} / \mathrm{o}$ responsables de la comunicación interna de cada empresa, o en su defecto quienes fungían como tales. Para determinar las características más importantes de este estudio se elaboró un análisis estadístico descriptivo. 


\section{Resultados}

La intencionalidad de la investigación, es la de conocer si las empresas preservan: cultura, clima, identidad e imagen. Se encontró que el $12,2 \%$ de las empresas encuestadas no poseen mecanismos de preservación de sus intangibles. El 27.27\%, afirma desarrollar mecanismos tanto para la identidad como para la imagen, el 18\% para el clima y el $15 \%$ para la cultura. Las empresas investigadas priorizan la identidad y la imagen por sobre el clima y la cultura.

\section{Tabla 1}

Medios para la difusión de acciones de preservación de intangibles

\begin{tabular}{lc}
\hline Medio de difusión & Empresas (\%) \\
\hline Newsletter & 2,00 \\
Blog corporativo & 6,00 \\
Correo electrónico & 24,00 \\
Intranet de la empresa & 18,00 \\
Página web de la empresa & 16,00 \\
Radio & 2,00 \\
Redes sociales & 24,00 \\
Revistas especializadas & 2,00 \\
Revistas internas & 4,00 \\
Televisión & 2,00 \\
\hline
\end{tabular}

Fuente: Elaboración propia.
En cuanto a si las empresas tienen medios a través de las cuales difunden acciones de preservación de sus intangibles, el $24 \%$ lo hacen principalmente por correo electrónico y redes sociales (Ver Tabla 1). En lo que respecta a actividades de difusión de acciones, el $17 \%$ realiza publicidad institucional, el $15 \%$ activaciones y un $12 \%$ eventos corporativos en general (Ver Tabla 2).

En cuanto a la satisfacción sobre la gestión de intangibles en las empresas, en la Tabla 3 se evidencia que

Tabla 2.

Actividades para la difusión de acciones de preservación de intangibles

\begin{tabular}{lc}
\hline \multicolumn{1}{c}{ Actividades de difusión } & Empresas (\%) \\
\hline Activaciones & 14,63 \\
Auspicios & 2,44 \\
Capacitaciones & 21,95 \\
Eventos corporativos en general & 12,20 \\
Marketing social & 9,76 \\
Mecenazgo & 2,44 \\
Ninguno de los anteriores & 2,44 \\
Patrocinio & 4,88 \\
Programas de Responsabilidad Social Corporativa & 7,32 \\
Publicidad Institucional & 17,07 \\
Publirreportajes & 4,88 \\
\hline
\end{tabular}

Fuente: Elaboración propia.

Tabla 3.

Satisfacción respecto de la gestión de intangibles (\% de empresas)

\begin{tabular}{lcccc}
\hline Nivel de satisfacción & $\begin{array}{c}\text { Cultura } \\
\text { organizaciona }\end{array}$ & $\begin{array}{c}\text { Clima } \\
\text { organizacional }\end{array}$ & $\begin{array}{c}\text { Gestión de la } \\
\text { identidad }\end{array}$ & $\begin{array}{c}\text { Gestión de la } \\
\text { imagen }\end{array}$ \\
\hline Totalmente insatisfecho & 11,11 & 5,56 & 5,56 & 5,56 \\
Muy insatisfecho & 5,56 & 0,00 & 0,00 & 0,00 \\
Bastante insatisfecho & 0,00 & 11,11 & 5,56 & 0,00 \\
Poco insatisfecho & 16,67 & 11,11 & 11,11 & 11,11 \\
Algo insatisfecho & 5,56 & 11,11 & 11,11 & 0,00 \\
Ni insatisfecho Ni & & & & \\
satisfecho & 27,78 & 33,33 & 22,22 & 22,22 \\
Algo satisfecho & 11,11 & 5,56 & 16,67 & 11,11 \\
Poco satisfecho & 16,67 & 11,11 & 5,56 & 16,67 \\
Bastante satisfecho & 0,00 & 5,56 & 16,67 & 5,56 \\
Muy satisfecho & 0,00 & 0,00 & 0,00 & 11,11 \\
Totalmente satisfecho & 5,56 & 5,56 & 5,56 & 16,67 \\
\hline
\end{tabular}

Fuente: Elaboración propia. 
aproximadamente 3 de cada 10 tienen una postura neutral.

Es evidente que, las empresas analizadas no preservan sus activos intangibles como deberían; muy al contrario de lo que idealmente deben hacer las empresas, y menos aún les dan a estos un enfoque o componente estratégico (Hinojosa y Cogco, 2020).

Acerca de si las empresas encuestadas poseen mecanismos en el que se estipulen políticas para su preservación, sucede que tan solo el $27 \%$ las posee en relación a la comunicación e imagen, en la misma porcentualidad la identidad corporativa, el $18 \%$ sobre clima organizacional y cultura en un $15 \%$. Con ello se demuestra que, siendo los intangibles una simbiosis obligada, las empresas en cuestión ignoran dicha sinergia y los gestionan de manera separada, situación que dista diametralmente con el marco teórico de esta investigación. Pues, si se considera que es la imagen la última beneficiaria, esta se asume posee una naturaleza de análisis multidimensional (De Castro y Navas, 2006).

En cuanto a los medios para la difusión de acciones para su preservación, las empresas encuestadas utilizan las redes, los correos electrónicos, el intranet y sitios web como los medios más idóneos (particular que se justificaría por la pandemia), y como actividades preponderantes para la difusión, las capacitaciones, la publicidad institucional, las activaciones y los eventos corporativos en general. Es notorio que la labor comunicativa es dispersa, no hay una estrategia de medios para tal efecto; no obstante, se reconoce los esfuerzos informativos de los sujetos investigados por darlos a conocer (Xifra, Ramos y Enríquez, 2016).

Sobre la satisfacción respecto de su gestión por parte de las empresas, los índices son bastante bajos, resultado que preocupa de sobremanera, y más si se toma en cuenta que la cultura:

Ha evolucionado de forma tal que actualmente no se puede separar cultura de organización, pues todas funcionan a través y dentro de ella, como resultado de una estructura social, por lo que las organizaciones deben considerar sus fortalezas culturales si desean adaptarse y sobrevivir a los cambios exigencias actuales y futuras. (Suárez et al., 2020, pp. 152-153).

Sobre el clima organizacional, al 33\% de los participantes ni le satisface ni le insatisface, porcentaje muy inferior en comparación con los análisis hechos en un estudio similar levantado en las principales cadenas de supermercados del Ecuador, en las cuales el clima tiene una aceptación de los colaboradores del 86,2\% (Paredes y Quiroz, 2021).

Con relación a la identidad, al 22,2\% de los entrevistados ni le satisface ni le insatisface, desconociendo con ello que un mensaje de identidad es parte de una imagen positiva en una organización, puesto que es a través de ella que se constituye una identidad de efecto interno, la cultura se comunica y la imagen se la proyecta, lo dicho es una de 
las conclusiones a las que llega Montaño (2019), en un estudio similar relacionado con indicadores para valorar la imagen de empresas del sector eléctrico en Esmeraldas.

Finalmente, y con respecto a la imagen al $22.2 \%$ de los directivos de comunicación ni le satisface ni le insatisface, así pasa inadvertida la imagen en sus cuatro niveles: imagen corporativa, productos y servicios, imagen interna e imagen institucional, elementos con los que se perfila la imagen real de toda organización (Fernández y Alarico, 2016).

\section{Conclusiones}

La investigación realizada refleja a groso modo la realidad actual de los intangibles. En respuesta a los mecanismos de preservación, medios $\mathrm{y}$ actividades que se llevan cabo para su difusión y sobre el grado de satisfacción que representa el esfuerzo de parte de las empresas, se concluye que mientras existe una amplia y dilatada bibliografía de la especialidad sobre intangibles, todo apunta a que las empresas van por carriles diferentes, y dejan al descubierto su intransigencia. La situación se agrava cuando son las empresas las que se encierran, no poseen políticas ni una cultura de preservación, siendo estos superados, en cuanto a importancia por los tangibles. No se duda de lo imprescindibles que estos son en el andamiaje de una empresa; sin ellos no hay empresa en el mundo que sobreviva, pero se debe entender que, junto con ellos, los intangibles también son relevantes.
Cuando se asevera que es la cultura organizacional el ADN de las empresas, se entiende que todas la tienen, en mayor o menor grado, es relegada a su suerte, y como efecto dominó, el resto de intangibles quedan sin sustento. A pesar de que el sentido común $\mathrm{y}$ las recomendaciones de los autores consultados aconsejan iniciar por la consolidación de la cultura, los resultados arrojan que son la identidad y la imagen las que demandan la atención y preocupación, lo que demuestra que las empresas no poseen un conocimiento amplio de su rol.

Al no poseer los mecanismos idóneos, las posibilidades de que los intangibles carezcan de preservación, es mayor. Su diligencia demanda la presencia de profesionales expertos y su perfil encuadra precisamente con un profesional formado en dirección de la comunicación, cuyo accionar se consolide sobre la base de una estrategia permanente en la que los medios, dependiendo de las circunstancias, estén programados, habiendo sido identificados con antelación.

Una empresa que asuma que el uso de ciertos canales comunicativos son los que la moda le impone, está destinada a sucumbir en su intento por salvaguardar su imagen. El uso de conductos electrónicos, y las redes sociales, son la consecuencia lógica de un mapeo previo de públicos, determinado por la idiosincrasia de su ecología mediática. En ello vemos un exacerbado uso de la web 2.0 que, sin restarle eficiencia, siempre dependerá de su oportunidad, no en vano 
la televisión sigue siendo un medio poderoso de disuasión.

Lo mismo que los medios, las tácticas en la preservación de los cuatro intangibles en cuestión, se derivan de una estrategia, estas no pueden ser determinadas a su libre albedrío, pues una acción comunicativa contiene el mensaje idóneo con el que debemos cumplir nuestros objetivos comunicacionales. El repentizar con las acciones comunicativas, traerá a la empresa daños irreversibles en cuanto a su imagen. Un ruido estructural se genera cuando el contenido no ha sido canalizado correctamente. Las capacitaciones son proactivas y parece ser que las empresas encuestadas las utilizan mucho, pero el dilema radica en su temporalidad. En esta encuesta se develó que la publicidad institucional es una actividad con frecuencia utilizada, pero como se sabe sus fines son más propagandísticos y no siempre responden a una realidad.

El definir procedimientos en el que consten las políticas de gestión de los intangibles es un gran paso en el camino por consolidar su preservación, el que las empresas investigadas cuenten con uno o dos es un avance; ahora bien, deben ser replanteados según las experiencias, pues sus acciones casi seguro quedarán obsoletas. Es una obligación actualizarlos permanentemente. $\mathrm{Si}$ bien existen estudios que acentúan y enfatizan indicadores de productividad, rentabilidad, rendimiento, eficiencia, entre otros aspectos, (Pinasco, 2019), para determinar el liderazgo de una empresa en su sector; en esa misma proporción, no hay reportes que evalúen los intangibles: clima, cultura, identidad, imagen y por añadidura la reputación de una organización. Son pocas las entidades que dedican su tiempo al levantamiento de esta información, tales como la AECI (2019). En tal virtud este aporte es imperativo, toda vez que mucho se habla de intangibles, pero escasas o inexistentes son las evidencias que demuestren su importancia.

Como limitaciones al presente estudio ha de referenciarse que se la llevó a cabo durante la pandemia, situación atípica para cualquier posible análisis, toda vez que las empresas priorizaron sus presupuestos para evitar o minimizar el impacto de la pandemia en sus operaciones, de ello se deduce que los intangibles no fueron prioridad, pero que paradójicamente deja al descubierto que, al no gestionarlos, la imagen de las empresas queda endeble y al descubierto de una potencial crisis. Además, vale precisar que, si bien la metodología permite alcanzar los objetivos específicos, permitiendo una primera aproximación, algunas cuestiones de interés precisarían estudios complementarios, como por ejemplo la correlación que hay entre la cultura y su incidencia en la constitución del clima, la identidad y la imagen.

Ciertamente hay estudios recientes hechos en el Ecuador en los que se analiza la correlación entre clima organizacional y desempeño laboral (Paredes y Quiroz, 2021), cultura corporativa y liderazgos (Falconí, 2020) e identidad e imagen (Vire, 2019); no 
obstante, no hay evidencia de estudios en los que se analice a estos cuatro intangibles de manera sinérgica y menos todavía si existe correlación alguna. Es así que este estudio podría ser el inicio para que, en futuras investigaciones, se viabilicen hacia el estudio de este fenómeno organizacional escasamente abordado.

En relación a la muestra y al instrumento con la ayuda de la aplicación Google Form, es necesario mencionar que los resultados de este estudio se levantaron en plena pandemia, razón por la cual no se hizo una investigación de campo. Sobre la transversalidad del estudio, sería interesante ensayar un examen longitudinal, toda vez que la pandemia trastocó el entorno corporativo, y las empresas como es lógico orientaron sus prioridades hacia sus tangibles y no hacia los intangibles.

\section{Contribución de autores}

R.D.R.G. Idea, revisión de literatura, y redacción de discusión y conclusiones.

W.G.N.A. Revisión de literatura, metodología y análisis de datos.

A.V.Z.A. Metodología y revisión de redacción.

\section{Referencias}

Aguirre, E., Calvache, C., y Osejo, D. (2019). Prueba Psicométrica MEIL: Motivación Extrínseca e Intrínseca en el Contexto Laboral. Revista Criterios, 26(2), 35-59. http://dx.doi.org/10.31948/ rev.criterios/26.2-art2
Asociación Ecuatoriana de Comunicación InternaAECI. (2019). Buenas prácticas de comunicación interna en el Ecuador. En F. Paladines y M. Abendaño (Eds), Colección de Comunicación Estratégica 2019. Aportes a la comunicación interna $y$ de crisis (138-163). Universidad Técnica Particular de Loja.

Brito, C., Pitre, R., y Cardona, D. (2020). Clima Organizacional y su Influencia en el Desempeño del Personal en una Empresa de Servicio. Información tecnológica, 31(1), 141-148. https://dx.doi.org/10. 4067/S0718-07642020000100141

Capriotti, P. (1999). Planificación Estratégica de Imagen Corporativa. Barcelona: Editorial Ariel.

Corresponsables. (2020). Monitor Empresarial de Reputación Corporativa (MERCO), Recuperado de https://ecuador.correspon sables.com/content/monitor-empresarialde-reputaci $\% \mathrm{C} 3 \% \mathrm{~B} 3 n$-corporativa-merco

De Castro, M., y Navas, J.E. (2006). La importancia de la reputación empresarial en la obtención de ventajas competitivas sostenibles. Investigaciones Europeas de Dirección y Economía de la Empresa, 12(3), 29-39.

Del Pozo, L. (2000). Gestión de la comunicación interna en las organizaciones, casos de empresas. Pamplona: Universidad de Navarra.

Falconí, F. (2020). Cultura corporativa y liderazgos locales en la Universidad Técnica de Cotopaxi. Revista de Ciencias Sociales y Humanidades Chakiñan, (12), 49-65. https://doi.org/10.37135/chk.002.12.03

Fernández, A., y Alarico, G.C. (2016). Evanescencia de la Imagen Corporativa: estrategias comunicativas de hoy. Venezuela: Universidad Católica Andrés Bello.

Garrido, F. (2008). Comunicación de la estrategia. 
La efectividad está en la dirección. Madrid - España: Ediciones DEUSTO.

Great Place To Work. (2020). Certifica la experiencia de tus empleados. Recuperado de https://www.greatplacetowork.com.ec/es/ servicios/certificacion

Hinojosa, L. J., y Cogco, C. A. (2020). Atractivo organizacional: Influencia de la Imagen y la Responsabilidad Social Corporativa. Revista de Psicología y Ciencias del Comportamiento de la Unidad Académica de Ciencias Jurídicas y Sociales, 11(1), 112-125. https://doi.org/ 10.29059/rpcc.20200617-106

Márquez, M. (2002, enero 30). Satisfacción laboral. Recuperado el 26 de julio de 2021, de https://www.gestiopolis.com/satisfaccion -laboral/

Molina, V. (2004). Imagen Corporativa. Ecuador: Gemagráfic.

Montaño, M. (2019). Indicadores para valorar la imagen pública externa del sector público en Esmeraldas. Revista Científica FIPCAEC, 4(11), 3-14. https://doi.org/10. 23857/fipcaec.v4i11.42

Paredes, P., y Quiroz, J. (2021). Correlación entre clima organizacional y desempeño laboral en las principales cadenas ecuatorianas de supermercados. Revista San Gregorio, (46), 83-95. http:// dx.doi.org/10.36097/rsan.v1i46.1746

Pinasco, G. (24 de octubre de 2019). Las 500 mayores empresas del Ecuador. Revista Vistazo. Recuperado de https://www. vistazo.com/seccion/pais/actualidad-naci onal/las-500-mayores-empresas-del-ecua dor

Ramos, D. (2019). Comunicación en las organizaciones: "cultura organizacional, modelo de competitividad de imprescindible gestión”. En F. Paladines y M. Abendaño (Eds), Colección de Comunicación
Estratégica 2019. Aportes a la comunicación interna y de crisis (94-96). Universidad Técnica Particular de Loja.

Ramos, D. (2012). La verdadera dimensión de la comunicación en las organizaciones. Bogotá: Ediciones San Pablo.

Ramos, E., y Valle, N. (2020). Gestión de imagen corporativa como estrategia de sostenibilidad: camino al cambio empresarial. Revista Universidad $y$ Sociedad, 12(1), 292-298.

Rekom, J., van and C.B.M. van Riel and B. Wierenga. (1991). Corporate Identity. Van vaag concept naar hard feitenmateriaal, working paper, Corporate Communication. Rotterdam: Centre: Erasmus University.

Scheinsohn, D. (1996). Comunicación estratégica, management y fundamentos de la imagen corporativa. Buenos Aires: Macchi Grupo Editor S.A.

Scheinsohn, D. (2009). Comunicación estratégica. Buenos Aires: Granica.

Sevilla, A. (17 de septiembre de 2015). Activo intangible. Economipedia. Recuperado el 25 de julio de 2021, de https:// economipedia.com/definiciones/activo-in tangible.html

Suárez, M., Álvarez, M., y Vásquez, M. (2020). La cultura organizacional a través de las teorías organizacionales: nacimiento, crecimiento y madurez. Revista San Gregorio, (40), 145-156. http://dx.doi. org/10.36097/rsan.v1i40.1387

Van Rekom, J., Van Riel, C.B.M., y Wierenga, B. (1991). Corporate Identity. Van vaag concept Naar Hard Feitenmateriaal, Rotterdam: Corporate Communication Centre, Erasmus University.

Van Riel, C. (1997). Comunicación corporativa. Madrid: Editorial Prentice Hall. 
Ventura, J. (2001). Comunicación Corporativa. En

J. Villafañe (Ed.), Dirección de comunicación empresarial e institucional (161-218). Barcelona: Gestión 2000.

Vire, J. (2019). Identidad e imagen corporativas. Relación entre los perfiles de identidad e imagen de la feria de Loja. Revista Internacional de Relaciones Públicas, 9(17),189-208. http://dx.doi.org/10.5783/ RIRP-17-2019-10-189-208.

Xifra, J., Ramos, D., y Enríquez, M. (2016). Teoría y Práctica de las Relaciones Públicas. Ecuador: UTE. 
